\title{
A Retrospective Cohort Study on the increasing trend of suicide ideations and risks in an opioid- dependent population of Puerto Rico 2015-2018
}

\section{Gerardo Collazo}

San Juan Bautista School of Medicine

\section{Alejandro Ruiz}

San Juan Bautista School of Medicine

\section{Marimer Rivera}

San Juan Bautista School of Medicine

Cristina Diaz-Marty

San Juan Bautista School of Medicine

\section{Alessandro Avila}

San Juan Bautista School of Medicine

Mauricio Escobar

San Juan Bautista School of Medicine

Juan Ojeda

San Juan Bautista School of Medicine

\section{Daniel Almodovar-Frau}

San Juan Bautista School of Medicine Irvin Maldonado

San Juan Bautista School of Medicine Yaritza Inostroza-Nieves ( $\sim$ yinostroza@sanjuanbautista.edu )

San Juan Bautista School of Medicine https://orcid.org/0000-0003-0359-2913

\section{Research Article}

Keywords: sex, poverty, unemployment status, past substance abuse, family characteristics, childhood traumas, personality traits, psychiatric diagnoses

Posted Date: September 23rd, 2021

DOI: https://doi.org/10.21203/rs.3.rs-503932/v1

License: (c) (1) This work is licensed under a Creative Commons Attribution 4.0 International License. Read Full License 
Version of Record: A version of this preprint was published at Journal of Immigrant and Minority Health on November 23rd, 2021. See the published version at https://doi.org/10.1007/s10903-021-01310-8. 


\section{Abstract}

Background: The rate of suicide attempts among people with substance abuse disorders in the U.S. is six times higher than in the general population. The prevalence of suicidal ideations and attempts continues to increase in Puerto Rico, with a significant incidence in substance-abusing populations.

Methods: This retrospective cohort study evaluate the suicide profile of 4,347 opioid-dependent participants in ASSMCA's methadone center in San Juan, PR, from 2015 to 2018 using questions related to suicidal ideation and attempts included in the admission questionnaire.

Results: Participants reporting suicide ideation increase from 8.5\% in 2015 to $17.0 \%$ in 2018. In 2015 only $7.0 \%$ claimed to have had a history of a suicide attempt, increasing to $12.4 \%$ in 2018 .

Discussion: Our data support the need for screening for suicide risk among substance-abusing populations to identify targeted interventions. The identification of high-risk populations for suicide can help during rehabilitation and finding the adequate resources needed.

\section{Introduction}

Suicide is the 10th leading cause of death in the United States (1). In 2019, 47,511 Americans died by suicide (1). Between 1999 and 2014, the annual age-adjusted death rate from suicide rates among opioid users in the United States has risen from 0.3 per 100,000 in 1999 to between 0.6 and 0.7 in 2014 (2). More than 1,300 deaths from suicide occurred in Puerto Rico from 2015 to 2020, estimating 8-25 attempts for every completed suicide (3). Suicide has become a leading cause of premature death in young adults.

The rate of suicide attempts among people with substance abuse disorders is six times higher than those who do not suffer from it, while heroin users are 14 times more likely to commit suicide $(4,5)$. Males with substance abuse disorders risk two to three times higher suicide, and females are 6.5 to 9 times higher than those who do not suffer from a substance abuse disorder. A 2011 study in Puerto Rico determined that polydrug users were significantly more likely to attempt suicide with an odds ratio of 8.8 (6).

Even though opioid overdose is the most common method of accidental death or suicide in substanceabusing populations, other methods, and risk factors are under study. Some include social and demographic status (sex, poverty, unemployment status, past substance abuse), family characteristics, childhood traumas, personality traits, and psychiatric diagnoses (depression, anxiety disorders, and suicidal ideations). Studies have concluded that suicide is a significant problem that treatment centers face, requiring targeted intervention if the rates among these groups decline (7).

In Puerto Rico, a government agency known as the Puerto Rico Administration of Mental Health and Antiaddiction Services (ASSMCA) has multiple functions. ASSMCA administers mental health treatment institutions and methadone centers, which are accessible, cost-effective, and confidence. They had six 
methadone centers (Bayamon, Caguas, Cayey, San Juan, Ponce and Aguadilla), two satellite units (Mayaguez and Arecibo) and three mobile units. The rehabilitation process (inpatient rehab, methadone center, medications, and support groups) increases the likelihood of recovery in these patients.

\section{Theoretical/conceptual Framework}

This study aimed to explore the prevalence of suicidal ideas and attempts in participants having an opioid substance abuse disorder upon entering treatment at a Methadone Center in San Juan, Puerto Rico.

\section{Methods}

This retrospective cohort study was designed to evaluate the suicide profile in opioid-dependent participants in ASSMCA's methadone center (CTIAM) in San Juan, PR, between January 1, 2015September 10, 2018 using the admission questionnaire.

\section{Participants}

During this period, 4,347 opioid-dependent participants filled out the admission questionnaire at San Juan's Comprehensive Drug-Assisted Treatment Clinic (Clinica de Tratamiento Integral Asistido con Medicamentos or CTIAM) a methadone center of ASSMCA. The requirements to participate in this clinic are 18 years of age or older, an opioid substance abuse disorder, and willingness to participate voluntarily.

\section{Data Collection and Analysis}

This study included all the participants who filled out the admission questionnaire from CTIAM in San Juan from January 1,2015 , to September 10,2018 . The clinic personnel interviewed the participants to answer the admission questionnaire, consisting of 45 questions and the DMS- 5 . The DMS-5 is an evaluation made by the clinic personnel, and the admission questionnaire had a space for the diagnostic code and name. Specific questions were selected from the admission questionnaire to obtain a profile of the participants who entered the program concerning suicide ideation and attempt. Those questions were: 1 . Gender 2. Age 3. Is there a history of suicidal ideas? 4. Is there a history of suicide attempts? 5. Have you participated in support groups, self-help, or religious meetings, or have you sought help from family, friends, or others during the past 30 days? These questions remain the same through the years of the study. ASSMCA provided us with the data grouped by year for these questions for analysis, and no patient medical record was used or seen.

The Institutional Review Board of San Juan Bautista School of Medicine approved this study (IRB ID\#: EMSJB-8-2018). Consent forms were not used, as there was no contact with subjects.

\section{Results}


The data analyzed represents 4,347 participants surveyed in the ASSMCA Methadone Center in San Juan, Puerto Rico, from 2015-2018. As shown in Table 1, of this total, $87 \%$ were male $(n=3,767)$, and $13 \%$ female $(n=580)$. The number of participants was classified per year $(n=1,154$ participants in $2015 ; n=$ 1,324 participants in 2016; $n=1,231$ in 2017 and $n=638$ participants in 2018). The data from the year 2018 was collected until September 10, 2018. Regarding age, we observed a trend for the mean age to decrease per year from $49.6( \pm 10.1)$ in 2015 to $47.8( \pm 10.1)$ in $2016,46.6( \pm 10.2)$ in 2017 , and $45.5( \pm$ 9.9) in 2018.

Table 1

Patient demographics.

\begin{tabular}{|lllll|}
\hline \multicolumn{5}{|c|}{ Year of Survey for Data Collection } \\
\hline & 2015 & 2016 & 2017 & 2018 \\
\hline Participants per year & 1154 & 1324 & 1231 & 638 * \\
\hline Gender & & & $176(14.3 \%)$ & $82(12.8 \%)$ \\
\hline Female & $142(12.3 \%)$ & $180(13.6 \%)$ & $1055(85.7 \%)$ & $556(87.2 \%)$ \\
\hline Male & $1012(87.7 \%)$ & $1144(86.4 \%)$ & $45.4( \pm 9.9)$ \\
\hline $\begin{array}{l}\text { Age } \\
\text { (Mean } \pm \text { Standard Deviation) }\end{array}$ & $49.6( \pm 10.1)$ & $47.8( \pm 10.1)$ & $46.6( \pm 10.2)$ & \\
\hline $\begin{array}{l}\text { Data was collected during a 12-month period during 2015-2017. *In 2018, data collection was } \\
\text { achieved until September 10, 2018. }\end{array}$ & & & \\
\hline
\end{tabular}

In 2015 , only one opioid user (0.09\%) reported participating in a support group over the past 30 days before answering the admission questionnaire. Participants reported receiving group support increased to $0.80 \%$ in $2016,4.20 \%$ in 2017 , and $4.20 \%$ in 2018 (data not shown).

Figure 1 shows that in $2015,8.5 \%$ of participants affirmed having ideations related to suicide, increasing to $10.6 \%$ in $2016,11.9 \%$ in 2017 , and $17.0 \%$ in 2018 . In 2015 only $7.0 \%$ claimed to have had a history of a suicide attempt, increasing to $8.5 \%$ in $2016,8.7 \%$ in 2017 , and $12.4 \%$ in 2018 .

\section{Discussion}

This study aimed to explore the prevalence of suicidal ideas and suicidal attempts in an opioiddependent population before entering a treatment and rehabilitation program at the CIATM Methadone Center in San Juan, Puerto Rico. We found that the prevalence of positive responses for suicide ideation was doubled from $2015(8.5 \%)$ to $2018(17 \%)$ in participants in a voluntary program to treat opioid abuse. The prevalence of positive participants for attempts to commit suicide increased from $7.0 \%$ in 2015 to $12.4 \%$ in 2018. 
Suicide, addiction, and psychiatric diagnosis have a close relationship and is well demonstrated in psychiatric and public health literature. More than $90 \%$ of patients presenting to the emergency department with suicidal ideation suffer from psychiatric diagnoses, have a substance abuse disorder, or both (8). Addiction frequently damages familial, professional, personal, and financial relationships, creating a dire cycle and increasing the risk of suicide.

In September 2017, Puerto Rico suffered a catastrophic natural disaster, which may be a variable impacting the increase of suicidal ideation and suicidal attempts observed in 2017 and 2018. Compared with 2016, the number of suicides in Puerto Rico 4 months after Hurricane Maria was $16 \%$ higher and $26 \%$ higher six months after the hurricane (9). There was an average of 19 suicides per month eight months before Hurricane Maria, which rose to an average of 25 suicides per month the three months following the hurricane (3). In 2018, a 6\% decrease in suicides in the general population living in Puerto Rico compared to 2017 (3). Contrary, we observed an increase in suicide ideation and attempts in 2018 in opioid-dependent participants. These increased rates found in the opioid-dependent participants could be attributable to multiple exposures, risks, and challenges more prevalent in this population as food insecurity, violent encounters, and fewer economic resources.

Despite the increase in suicidal ideations and attempts, we noticed an increasing trend to search for help in support groups. Participation in support groups increases from $0.09 \%$ in 2015 to $4.20 \%$ in 2018 . Support groups positively impact participants' self-efficacy, increased satisfaction with the treatment experience, reduced relapse rates, and increased retention in treatment (10). Support groups can provide a practical approach to help opioid-dependent participants strive to achieve and maintain recovery.

Since the use of opioids in suicides has been increasing over time, our findings emphasize the need for health care providers to assess suicidal risk in patients receiving methadone as treatment. This increase in suicide ideations and attempts in opioid-dependent participants looking for help is especially crucial in rehabilitation centers to target appropriate interventions, and management approaches to recognize those at risk and prevent suicide.

Our study has some limitations, such as that the retrospective data that was analyzed is from one methadone center (San Juan) out of six centers in Puerto Rico, and the data collected from the year 2018 was not from the entire year in contrast with the data from 2015, 2016, and 2017. Nevertheless, our data provide further evidence that suicide risk increases in the opioid drug-abusing population in Puerto Rico.

\section{Conclusion}

In conclusion, we found an increase in suicidal ideations and attempts in the opioid-dependent participants from 2015 to 2018. It is unclear what is driving these changes and how they might be related to one another. Nevertheless, in 2017, Puerto Rico was devasted by Hurricane Maria, increasing suicide rates among the Puerto Rican population. In 2018, suicide rates in the general population decrease. However, the opioid-dependent participants are a vulnerable population with difficulty finding food and clothing, property loss, and economic problems contributing to suicide ideation. Our data support the 
need for screening for suicide risk among those who use illicit substances to identify targeted interventions. The psychological aspect is essential to improve patient outcomes in a detox center and decrease suicidal ideation and attempts. Support groups could be a promising strategy to guide opioiddependent participants through recovery.

\section{Declarations}

\section{New Contribution to Literature}

To our knowledge, this descriptive analysis of suicidal ideation and suicidal attempts among a cohort of the opioid-dependent population seeking methadone treatment and rehabilitation is the first study linking suicide risk and opioid use among a Puerto Rican sample. The consideration of suicide risk factors by different treatment centers can help administer better treatment to their participants. They can support and encourage their participants to overcome traumas, physical and mental health-related issues that may be causing them to think of suicide as a viable option to solve their situations.

\section{Acknowledgments}

We want to thank the "Administración de Servicios de Salud Mental y Contra la Adicción" also known as ASSMCA, and the Methadone Treatment Center (CTIAM), a sub-branch of ASSMCA in San Juan, Puerto Rico for providing the data. We also want to thank Dr. Estela S. Estape for her coaching during the writing of this manuscript.

\section{References}

1. Centers for Disease Control and Prevention, National Center for Health Statistics. Underlying Cause of Death 1999-2019 on CDC WONDER Online Database, released in 2020. Data are from the Multiple Cause of Death Files, 1999-2019, as compiled from data provided by the 57 vital statistics jurisdictions through the Vital Statistics Cooperative Program. Accessed at http://wonder.cdc.gov/ucd-icd10.html

2. Braden, J. B., Edlund, M. J., \& Sullivan, M. D. (2017). Suicide deaths with opioid poisoning in the United States: 1999-2014. American journal of public health, 107(3), 421-426.

3. Coss Guzmán, M. I. \& Román Vázquez, N. I. (2021) Informe Mensual De Suicidios En Puerto Rico. Accessed at http://www.salud.gov.pr/Estadisticas-Registros-yPublicaciones/Estadisticas\%20Suicidio/2021/Enero\%202021.pdf

4. Dragisic, T., Dickov, A., Dickov, V., \& Mijatovic, V. (2015). Drug addiction as risk for suicide attempts. Materia socio-medica, 27(3), 188-191.

5. Darke, S., \& Ross, J. (2002). Suicide among heroin users: rates, risk factors and methods. Addiction, 97(11), 1383-1394.

6. Reyes, J. C., Robles, R. R., Colón, H. M., Negrón, J. L., Matos, T. D., \& Calderón, J. M. (2011). Polydrug use and attempted suicide among Hispanic adolescents in Puerto Rico. Archives of suicide research, 
15(2), 151-159.

7. Gaynes, B. N., West, S. L., Ford, C. A., Frame, P., Klein, J., \& Lohr, K. N. (2004). Screening for suicide risk in adults: a summary of the evidence for the US Preventive Services Task Force. Annals of internal medicine, 140(10), 822-835.

8. Allison Tadros, Melinda Sharon, Michael Crum, Ryan Johnson, Kimberly Quedado, Wei Fang. (2020). Coexistence of Substance Abuse among Emergency Department Patients Presenting with Suicidal Ideation. BioMed Research International, vol. 2020, Article ID 7460701, 5 pages.

9. Ramphal L. (2018). Medical and psychosocial needs of the Puerto Rican people after Hurricane Maria. Proceedings (Baylor University. Medical Center), 31(3), 294-296.

10. Tracy, K., \& Wallace, S. P. (2016). Benefits of peer support groups in the treatment of addiction. Substance abuse and rehabilitation, 7, 143-154.

\section{Figures}

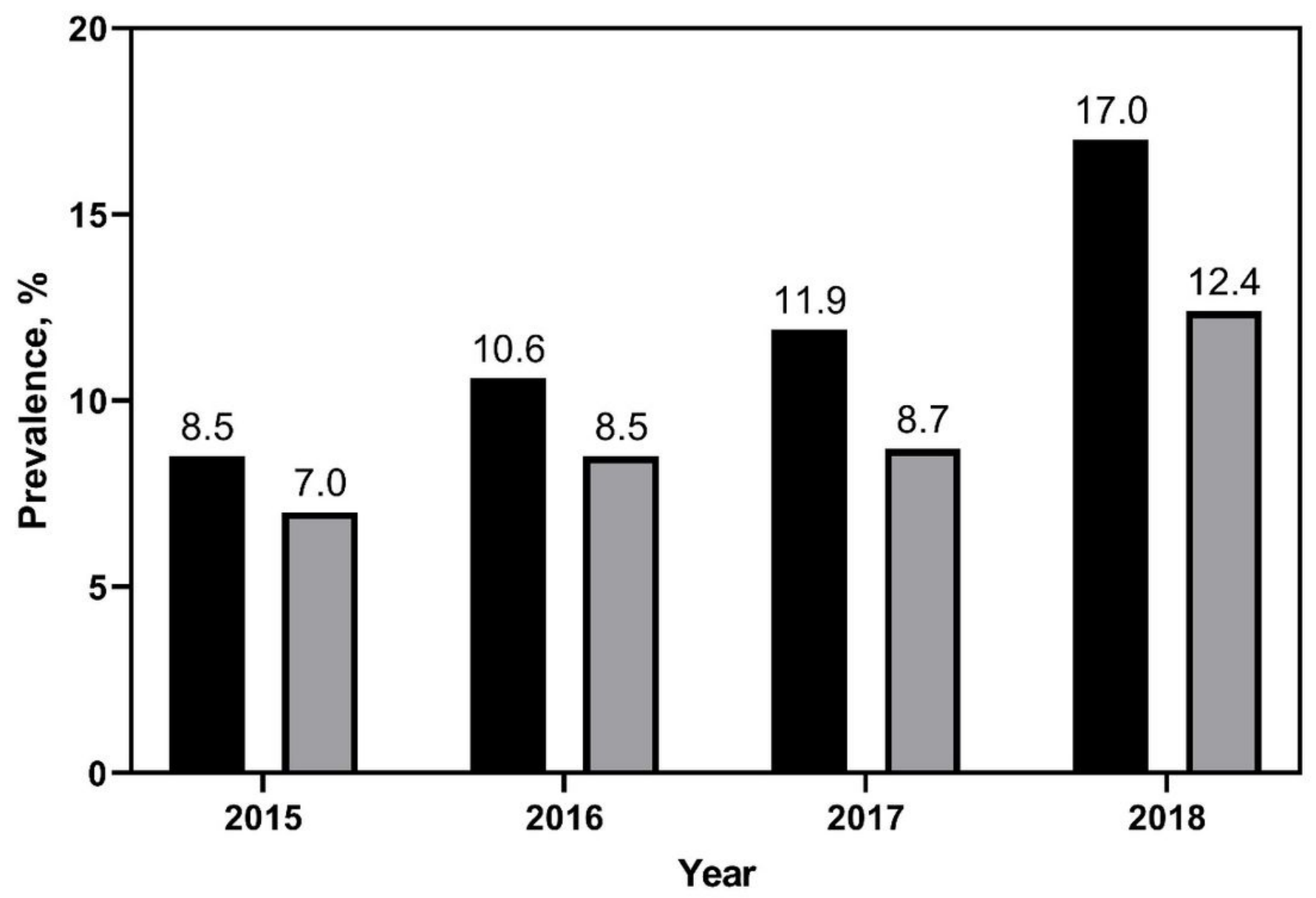

m Suicide Ideation $\square$ Suicide Attempts

Figure 1 
Prevalence of Suicidal Ideation and Suicidal Attempts. Suicidal Ideation and Attempts of the 4,347 participants surveyed in the ASSMCA Methadone Center in San Juan, Puerto Rico, from 2015-2018. 